\title{
A NEW DELAYED FEEDBACK CONTROL SCHEME FOR DISCRETE TIME CHAOTIC SYSTEMS
}

\author{
Ömer Morgül \\ Bilkent University, Dept. of Electrical and Electronics \\ Engineering, 06800, Bilkent, Ankara, Turkey
}

\begin{abstract}
In this paper we consider the stabilization problem of unstable periodic orbits of discrete time chaotic systems. We consider both one dimensional and higher dimensional cases. We propose a novel generalization of the classical delayed feedback law and present some stability results. These results show that for period 1 all hyperbolic periodic orbits can be stabilized with the proposed method. Although for higher order periods the proposed scheme may possess some limitations, some improvement over the classical delayed feedback scheme still can be achieved with the proposed scheme. The stability proofs also give the possible feedback gains which achieve stabilization. We will also present some simulation results.
\end{abstract}

Keywords: Chaotic Systems, Chaos Control, Delayed Feedback System, Pyragas Controller, Stability.

\section{INTRODUCTION}

Many physical systems may be represented by mathematical models which exhibit chaotic behaviour, see e.g. (Chen and Dong, 1999). Hence in recent years, various aspects of chaotic systems have received considerable interest. Due to possible applications, the subject of controlling chaos has also attracted a great deal of attention, see e.g. (Chen and Dong, 1999), and the references therein.

Chaotic systems may possess many unstable periodic orbits and usually these orbits are embedded in some strange attractors. Although one may define various control problems for chaotic systems, one of the interesting problems is to find some control schemes to achieve the stabilization of some of these periodic orbits. If this is achieved, such control schemes may force the chaotic systems to exhibit regular behaviour, see e.g. (Chen and Dong, 1999). A remarkable result first given in (Ott, Grebogy and Yorke, 1990) shows that by using small external forces it may be possible to stabilize some of these orbits. Following the semi- nal work of (Ott, Grebogy and Yorke, 1990), various other control methods have been proposed for the cited problem. Among these, the Delayed Feedback Control (DFC) scheme first proposed in (Pyragas, 1992) has received attention due to its various attractive features. This scheme has also been used in various applications, see e.g. (Pyragas, 2001), (Morgül, 2003), (Morguil, 2006), and the references therein. As it is shown in (Morgül, 2003), (Ushio, 1996), (Nakajima, 1997), (Morgül, 2005a), the classical DFC has certain inherent limitations, i.e. it cannot stabilize certain periodic orbits. We note that a recent result presented in (Fiedler et al., 2007), showed clearly that under certain cases, odd number limitation property does not hold for autonomous continuous time system. Although the subject is still open and deserves further investigation, we note that the limitation of DFC stated above holds for discrete time case, see e.g. (Ushio, 1996), (Morgül, 2003), (Morgül, 2005a).

To overcome the limitations of classical DFC scheme, various modifications have been proposed, see e.g. (Pyragas, 2001), (Socolar et. al., 1994), (Pyragas, 
1995), (Bleich, and Socolar, 1996), (Vieira, and Lichtenberg, 1996), and the references therein. One of these schemes is the so-called periodic, or oscillating feedback, and is known that it eliminates the limitations of classical DFC for period $\mathrm{T}=1$ case. This scheme can be generalized to the case $T>1$ in various ways, and two such generalizations are given in (Morgül, 2006), (Morgül, 2005b) ; it has been shown in these references that any hyperbolic periodic orbit can be stabilized with these schemes. Another modification is the so-called extended DFC (EDFC), see (Socolar et. al., 1994). This scheme is then analyzed and various of its modifications have been proposed, see e.g. (Pyragas, 2001), (Pyragas, 1995), (Bleich, and Socolar, 1996), (Vieira, and Lichtenberg, 1996), and the references therein. It has also been shown that EDFC also has inherent limitations similar to the DFC. In (Vieira, and Lichtenberg, 1996), a nonlinear version of EDFC has been proposed and it was shown that an optimal version of this scheme becomes quite simple. In this paper we will propose a scheme which is related to the optimal control proposed in (Vieira, and Lichtenberg, 1996) for one dimensional systems for the case $T=1$. We then generalize the proposed scheme for multi-dimensional case and for $T>1$. Our approach is similar to the one used in (Morgül, 2009), where only one dimensional discrete time chaotic systems were considered.

This paper is organized as follows. In section 2 we will outline the basic problem. In section 3 we will propose a new generalization of the DFC scheme and provide some stability results. In section 4 we will extend these results for higher dimensional case. In section 5 we will provide some simulation results and finally we will give some concluding remarks.

\section{PROBLEM STATEMENT}

Let us consider the following discrete-time system

$$
x(k+1)=f(x(k)),
$$

where $k=1,2 \ldots$ is the discrete time index, $x \in \mathbf{R}^{n}, f$ : $\mathbf{R}^{n} \rightarrow \mathbf{R}^{n}$ is an appropriate function, which is assumed to be differentiable wherever required. We assume that the system given by (1) possesses a period $T$ orbit characterized by the set

$$
\Sigma_{T}=\left\{x_{1}^{*}, x_{2}^{*}, \ldots, x_{T}^{*}\right\},
$$

where $x_{i}^{*} \in \mathbf{R}^{n}, i=1,2, \ldots, T$.

Let $x(\cdot)$ be a solution of (1). To characterize the convergence of $x(\cdot)$ to $\Sigma_{T}$, we need a distance measure, which is defined as follows. For $x_{i}^{*}$, we will use circular notation, i.e. $x_{i}^{*}=x_{j}^{*}$ for $i=j(\bmod (T))$. Let us define the following indices $(j=1, \ldots, T)$ :

$$
d_{k}(j)=\sqrt{\sum_{i=0}^{T-1}\left\|x(k+i)-x_{i+j}^{*}\right\|^{2}},
$$

where $\|\cdot\|$ is the standard Euclidean norm on $\mathbf{R}^{n}$.

We then define the following distance measure

$$
d\left(x(k), \Sigma_{T}\right)=\min \left\{d_{k}(1), \ldots, d_{k}(T)\right\} .
$$

Clearly, if $x(1) \in \Sigma_{T}$, then $d\left(x(k), \Sigma_{T}\right)=0, \forall k$. Conversely if $d\left(x(k), \Sigma_{T}\right)=0$ for some $k_{0}$, then it remains 0 and $x(k) \in \Sigma_{T}$, for $k \geq k_{0}$. We will use $d\left(x(k), \Sigma_{T}\right)$ as a measure of convergence to the periodic solution given by $\Sigma_{T}$.

Let $x(\cdot)$ be a solution of (1) starting with $x(1)=x_{1}$. We say that $\Sigma_{T}$ is (locally) asymptotically stable if there exists an $\varepsilon>0$ such that for any $x(1) \in \mathbf{R}^{n}$ for which $d\left(x(1), \Sigma_{T}\right)<\varepsilon$ holds, we have $\lim _{k \rightarrow \infty} d\left(x(k), \Sigma_{T}\right)=$ 0 . Moreover if this decay is exponential, i.e. the following holds for some $M \geq 1$ and $0<\rho<1,(k>1)$

$$
d\left(x(k), \Sigma_{T}\right) \leq M \rho^{k} d\left(x(1), \Sigma_{T}\right),
$$

then we say that $\Sigma_{T}$ is (locally) exponentially stable.

To stabilize the periodic orbits of (1), let us apply the following control law :

$$
x(k+1)=f(x(k))+u(k)
$$

where $u(\cdot) \in \mathbf{R}^{n}$ is the control input. In classical DFC, the following feedback law is used $(k>T)$ :

$$
u(k)=K(x(k)-x(k-T)),
$$

where $K \in \mathbf{R}^{n \times n}$ is a constant gain to be determined. It is known that the scheme given above has certain inherent limitations, see e.g. (Ushio, 1996). For simplicity, let us assume one dimensional case, i.e. $n=1$. For $\Sigma_{T}$, let us set $a_{i}=f^{\prime}\left(x_{i}^{*}\right)$. It can be shown that $\Sigma_{T}$ cannot be stabilized with this scheme if $a=\prod_{i=1}^{T} a_{i}>1$, see e.g. (Morgül, 2003), (Ushio, 1996), and a similar condition can be generalized to the case $n>1$, (Nakajima, 1997), (Morgül, 2005a). A set of necessary and sufficient conditions to guarantee exponential stabilization can be found in (Morgül, 2003) for $n=1$ and in (Morgül, 2005a) for $n>1$. By using these results one can find a suitable gain $K$ when the stabilization is possible.

\section{A NOVEL GENERALIZATION OF DFC}

As mentioned in the introduction, to overcome the basic limitations of the classical DFC various modifications has been proposed in the literature . Among these, for one dimensional case (i.e. $n=1$ ), the EDFC scheme first proposed in (Socolar et. al., 1994) and its nonlinear version proposed in (Vieira, and Lichtenberg, 1996) deserve special attention. In the sequel, first we will consider one dimesional case $(n=1)$ and propose a scheme which is related to the optimal version of the scheme proposed in (Vieira, and Lichtenberg, 1996) for the period 1 case. Then we propose 
a novel generalization of this scheme for higher order periods and higher dimensional case. For the details of our approach for one dimensional case, see (Morgül, 2009). Later we will generalize this approach to higher dimensional case, which is not considered in (Morgül, 2009).

To motivate our approach, we first consider the one dimensional case, i.e. $n=1$ throughout this section. For simplicity, let $\Sigma_{1}=\left\{x_{1}^{*}\right\}$ be a period 1 orbit of (1) (i.e. fixed point of $f: \mathbf{R} \rightarrow \mathbf{R}$ ), and consider the controlled system given by (6). Instead of the DFC scheme given by (7), let us propose the following law

$$
u(k)=\frac{K}{K+1}(x(k)-f(x(k))
$$

where $K \in \mathbf{R}$ is a constant gain to be determined. Clearly we require $K \neq-1$. By using (8) in (6), we obtain :

$$
x(k+1)=\frac{1}{K+1} f(x(k))+\frac{K}{K+1} x(k) .
$$

Obviously on $\Sigma_{1}$, we have $u(k)=0$, see (8). Furthermore if $x(k) \rightarrow \Sigma_{1}$ (i.e. when $\Sigma_{1}$ is asymptotically stable) we have $u(k) \rightarrow 0$ as well. Therefore, the scheme proposed in (8) enjoys the similar properties of DFC.

Remark 1 : The scheme given by (8)has an interesting relation with the classical DFC scheme. To see that, if we multiply (9) with $K+1$, after simplification we obtain :

$$
x(k+1)=f(x(k))+K(x(k)-x(k+1))
$$

If we compare (10) with (6), we see that they become similar if we use the following equation for $u(k)$ :

$$
u(k)=K(x(k)-x(k+1)) .
$$

However, this is only a mathematical similarity since $u(k)$ given by (11) is not implementable as a control law. Nevertheless, at least from mathematical point of view, (11) shows an interesting connection between the classical DFC and the scheme proposed in this paper.

Next, we will consider the stability of $\Sigma_{1}$ as defined in the section 2. For simplicity, set $\Sigma_{1}=\left\{x_{1}^{*}\right\}, a=a_{1}=$ $f^{\prime}\left(x_{1}^{*}\right)$. By using linearization, (9) and the classical Lyapunov stability analysis, we can easily show that $\Sigma_{1}$ is exponentially stable for (9) if and only if

$$
\left|\frac{K+a}{K+1}\right|<1 \text {. }
$$

It can easily be shown that if $a \neq 1$, then any $\Sigma_{1}$ can be stabilized by choosing $K$ appropriately to satisfy (12). In fact, for any $\rho$ satisfying $-1<\rho<1$, we can choose the stabilizing gain as :

$$
K=\frac{\rho-a}{1-\rho}
$$

Hence the limitations of DFC and EDFC are eliminated greatly by the proposed approach. It appears that the only restriction remains (i.e. $a \neq 1$ ) is quite inherent and appears in (Morgül, 2006) and (Morgül, $2005 \mathrm{~b}$ ) as well. By using the arguments given in these latter references, we can state that all hyperbolic fixed points can be stabilized with the proposed scheme.

At this point we can generalize the control law given by (9) to $T=m$ case. By following the ideas given above, we propose the following control law :

$$
u(k)=\frac{K}{K+1}(x(k-m+1)-f(x(k)),
$$

where $K \in \mathbf{R}$ is a constant gain to be determined. If we use (14) in (6), we obtain :

$$
x(k+1)=\frac{1}{K+1}(f(x(k))+K x(k-m+1)) .
$$

Remark 2 : As mentioned in Remarks 1 , the scheme given above has an interesting relation with the classical DFC scheme. To see that, if we multiply (15) with $K+1$, after simplification we see that (15) is similar to (6), if we use the following equation for $u(k)$ :

$$
u(k)=K(x(k-m+1)-x(k+1)) .
$$

However, this is only a mathematical similarity since $u(k)$ given by (16) is not implementable as a control law.

For stability analysis, we will follow the methodology given in (Morgül, 2003), (Morgül, 2005a). As before, let us define $x_{i}(k)=x(k-m+i), i=1,2, \ldots, m$ and $z=\left(x_{1} \ldots x_{m}\right)^{T}$. Let us define

$$
\begin{aligned}
& Y_{i}=\frac{1}{K+1} f\left(Y_{i-1}\right)+\frac{K}{K+1} x_{i} \\
& Y_{0}=x_{m}, i=1,2, \ldots, m \text {. }
\end{aligned}
$$

Let us define the map $F: \mathbf{R}^{m} \rightarrow \mathbf{R}^{m}$ as $F(z)=$ $\left(x_{2} x_{3} \ldots x_{m} Y_{1}\right)^{T}$. Clearly we have $F^{m}=\left(Y_{1} Y_{2} \ldots Y_{m}\right)^{T}$. Now, consider the map

$$
z(k+1)=F^{m}(z(k)) .
$$

Now consider the fixed points of (18), i.e. $F^{m}\left(z^{*}\right)=z^{*}$ where $z^{*}=\left(x_{1}^{*} x_{2}^{*} \ldots x_{m}^{*}\right)^{T}$.Clearly we will have $x_{i}^{*}=Y_{i}$ where $i=1,2, \ldots, m$ and $Y_{i}$ are given by (17). Solving these equations we easily obtain $x_{i+1}^{*}=f\left(x_{i}^{*}\right), i=$ $1,2, \ldots, m-1$ and $x_{1}^{*}=f\left(x_{m}^{*}\right)$. This shows that a fixed point $z^{*}$ of (18) corresponds to a period $m$ orbit $\Sigma_{m}$ of (1), and vice versa. Therefore for the stability of $\Sigma_{m}$, we can study the stability of the fixed point $z^{*}$ of (18). This can be done by standard linearization, i.e. by finding the Jacobian $J_{m}=\frac{\partial F^{m}}{\partial z} \mid \Sigma_{m}$. Clearly we have $J_{m}(i, j)=\frac{\partial Y_{i}}{\partial x_{j}} \mid \Sigma_{m}$. By using (17), after straightforward calculations we obtain : 


$$
\begin{aligned}
& \frac{\partial Y_{i}}{\partial x_{i}}= \frac{K}{K+1}, i=1,2, \ldots, m-1 \\
&, \frac{\partial Y_{m}}{\partial x_{m}}=\frac{K}{K+1}+\frac{a}{(K+1)^{m}} \\
& \frac{\partial Y_{i}}{\partial x_{j}}=\frac{a_{i-1}}{K+1} \frac{\partial Y_{i-1}}{\partial x_{j}} \\
&, i, j=1,2, \ldots, m, i \neq j
\end{aligned}
$$

where by convention we have $a_{0}=a_{m}, Y_{0}=Y_{m}$. For stability analysis, we need the characteristic polynomial of $J_{m}$, which is given in the following Theorem.

Theorem 1 : Let $\Sigma_{m}$ given by (2) be a period $T=m$ orbit of (1) and set $a_{i}=f^{\prime}\left(x_{i}\right), i=1,2, \ldots, m, a=$ $\prod_{i-1}^{m} a_{i}$. Consider the Jacobian $J_{m}$ given by (17)-(20). Then for $m \geq 1$ we have :

$$
p_{m}(\lambda)=\left(\lambda-\frac{K}{K+1}\right)^{m}-\frac{a}{(K+1)^{m}} \lambda^{m-1}
$$

Proof : This result can easily be shown either by using direct calculation of $\operatorname{det}\left(\lambda I-J_{m}\right)=$, where $I$ is an identity matrix with appropriate dimensions, or by using mathematical induction. The calculations are straightforward but rather lengthy and hence are omitted here.

We say that a polynomial is Schur stable if all of its roots are inside the unit disc of the complex plane, i.e. have magnitude less than unity. Hence the asymptotic stability of the fixed points of (18) hence the asymptotic stability of $\Sigma_{m}$ for (6) and (14) could be analyzed by considering the Schur stability of $p_{m}(\lambda)$. Moreover note that the exponential stability is equivalent to Schur stability, see (Khalil, 2002). By using these, we can state our next result.

Theorem 2 : Let $\Sigma_{m}$ given by (2) be a period $T=m$ orbit of (1) and set $a_{i}=f^{\prime}\left(x_{i}\right), i=1,2, \ldots, m, a=$ $\prod_{i-1}^{m} a_{i}$. Consider the control scheme given by (6) and (14). Then :

i : $\Sigma_{m}$ is exponentially stable if and only if $p_{m}(\lambda)$ given by (21) is Schur stable. This condition is only sufficient for asymptotic stability.

ii : If $p_{m}(\lambda)$ has at least one unstable root, i.e. outside the unit disc, then $\Sigma_{m}$ is unstable as well.

iii : If $p_{m}(\lambda)$ is marginally stable, i.e. has at least one root on the unit disc while the rest of the roots are inside the unit disc, then the proposed method to test the stability of $\Sigma_{m}$ is inconclusive.

Proof : The proof of this Theorem easily follows from standard Lyapunov stability arguments, see e.g. (Khalil, 2002), and (Morgül, 2003), (Morgül, 2005a) for similar arguments.

\section{HIGHER DIMENSIONAL CASE}

The scheme given above can be easily generalized to higher dimensional case (i.e. $n>1$ ). However, as will be shown below, the conclusions may not be as simple as one dimensional case.

To motivate the analysis, let us consider the case $T=$ 1. More precisely, let $\Sigma_{1}=\left\{x_{1}^{*}\right\}$, where $x_{1}^{*} \in \mathbf{R}^{n}$ be a period 1 orbit of (1) (i.e. fixed point of $f: \mathbf{R}^{n} \rightarrow$ $\mathbf{R}^{n}$ ), and consider the controlled system given by (6). Instead of the DFC scheme given by (7), let us propose the following law :

$$
u(k)=(K+I)^{-1} K(x(k)-f(x(k)),
$$

where $K \in \mathbf{R}^{n \times n}$ is a constant gain matrix to be determined, and $I$ is $n \times n$ identity matrix. Clearly, we require that $K$ does not have an eigenvalue -1 . By using (22) in (6), we obtain :

$$
x(k+1)=(K+I)^{-1}(f(x(k))+K x(k)) .
$$

Obviously on $\Sigma_{1}$, we have $u(k)=0$, see (22). Furthermore if $x(k) \rightarrow \Sigma_{1}$ (i.e. when $\Sigma_{1}$ is asymptotically stable) we have $u(k) \rightarrow 0$ as well. Therefore, the scheme proposed in (8) enjoys the similar properties of DFC.

Remark 3 : The scheme given by (22)has an interesting relation with the classical DFC scheme. To see that, if we multiply (23) with $K+I$, after simplification we obtain :

$$
x(k+1)=f(x(k))+K(x(k)-x(k+1)) .
$$

If we compare (24) with (6), we see that they become similar if we use the following equation for $u(k)$ :

$$
u(k)=K(x(k)-x(k+1)) .
$$

However, this is only a mathematical similarity since $u(k)$ given by (25) is not implementable as a control law. Nevertheless, at least from mathematical point of view, (25) shows an interesting connection between the classical DFC and the scheme proposed in this paper. See also remarks 1 and 2 .

Next, we will consider the stability of $\Sigma_{1}$ as defined in the section 2. For simplicity, set $\Sigma_{1}=\left\{x_{1}^{*}\right\}, J=$ $J_{1}=\left.\frac{\partial f}{\partial x}\right|_{x=x_{1}^{*}}$. By using linearization, (23) and the classical Lyapunov stability analysis, we can easily show that $\Sigma_{1}$ is exponentially stable for (23) if and only if $(K+I)^{-1}(J+K)$ is a Schur stable matrix. To see the limitation of our approach, similar to the one dimensional case, assume that $J$ has an eigenvalue 1 . Let $\xi$ be the corresponding eigenvector, i.e. $J \xi=\xi$. Then we have $(K+I)^{-1}(J+K) \xi=(K+I)^{-1}(I+$ $K) \xi=\xi$. Hence, if $J$ has an eigenvalue 1 , so is the matrix $(K+I)^{-1}(J+K)$ for any $K$. Therefore, if $J$ has an eigenvalue 1 , exponential stabilization is not possible. Otherwise, by choosing an appropriate $K$, one can always stabilize $\Sigma_{1}$. More precisely, let $\Lambda$ be any Schur stable matrix. Then $K=(I-\Lambda)^{-1}(\Lambda-J)$ will stabilize $\Sigma_{1}$, see (13). This result shows that the limitations of DFC and EDFC are eliminated greatly 
by the proposed approach. Hence, as in the one dimensional case, we can state that all hyperbolic fixed points can be stabilized with the proposed scheme.

To proceed, let us consider the case $T=2$, in which case we propose the following control law :

$$
u(k)=(K+I)^{-1} K(x(k-1)-f(x(k)) .
$$

If we use (26) in (6), we obtain :

$$
x(k+1)=(K+I)^{-1}(f(x(k))+K x(k-1)) .
$$

Remark 4 : The scheme given by (26)has an interesting relation with the classical DFC scheme. To see that, if we multiply (27) with $K+I$, after simplification we obtain :

$$
x(k+1)=f(x(k))+K(x(k-1)-x(k+1)) .
$$

If we compare (28) with (6), we see that they become similar if we use the following equation for $u(k)$ :

$$
u(k)=K(x(k-1)-x(k+1)) \text {. }
$$

However, this is only a mathematical similarity since $u(k)$ given by (29) is not implementable as a control law. Nevertheless, at least from mathematical point of view, (29) shows an interesting connection between the classical DFC and the scheme proposed in this paper. See also remarks 1, 2 and 3 .

Let $\Sigma_{2}=\left\{x_{1}^{*}, x_{2}^{*}\right\}$ be a period 2 orbit of (1) and let us set

$$
J_{1}=\left.\frac{\partial f}{\partial x}\right|_{x=x_{1}^{*}}, \mathbf{J}_{2}=\left.\frac{\partial f}{\partial x}\right|_{x=x_{2}^{*}}, \quad \mathbf{J}=\mathbf{J}_{1} J_{2} .
$$

For stability analysis, we will follow the methodology given in (Morgül, 2003), (Morgül, 2005a). Let us define $x_{1}(k)=x(k-1), x_{2}(k)=x(k)$ and $z=\left(x_{1} x_{2}\right)^{T}$ where the superscript $T$ denotes the transpose. Let us define a map $F: \mathbf{R}^{2 n} \rightarrow \mathbf{R}^{2 n}$ as $F(z)=\left(x_{2} Y_{1}\right)^{T}$ where $Y_{1}=(K+I)^{-1} f\left(x_{2}\right)+(K+I)^{-1} K x_{1}$. Clearly we have $F^{2}(z)=\left(\begin{array}{ll}Y_{1} & Y_{2}\end{array}\right)^{T}$ where $Y_{2}=(K+I)^{-1} f\left(Y_{1}\right)+(K+$ $I)^{-1} K x_{2}$. Let us consider the system :

$$
z(k+1)=F^{2}(z(k)) .
$$

Consider the fixed points of (31), i.e $F^{2}\left(z^{*}\right)=z^{*}$ where $z^{*}=\left(x_{1}^{*} x_{2}^{*}\right)^{T}$. Solving the fixed point equation, after simple calculations we obtain $x_{2}^{*}=f\left(x_{1}^{*}\right)$ and $x_{1}^{*}=f\left(x_{2}^{*}\right)$. Hence the fixed point $z^{*}$ of (31) corresponds to a period 2 orbit $\Sigma_{2}$ of (1), and vice versa. Therefore for the stability of $\Sigma_{2}$, we study the stability of the corresponding fixed point $z^{*}$ for the map $F^{2}$. This can be done by standard linearization. The Jacobian $J_{F}=\frac{\partial F^{2}}{\partial z} \mid \Sigma_{2}$ can easily be obtained as :

$$
J_{F}=\left[\begin{array}{ll}
J_{11} & J_{12} \\
J_{21} & J_{22}
\end{array}\right] \text {. }
$$

where $J_{11}=(K+I)^{-1} K, J_{12}=(K+I)^{-1} J_{2}, J_{21}=$ $(K+I)^{-1} J_{1}(K+I)^{-1} K, J_{22}=(K+I)^{-1} K+(K+$
$I)^{-1} J_{1}(K+I)^{-1} J_{2}$. We can clearly state that $\Sigma_{2}$ is exponentially stable if and only if $J_{F}$ given above is a Schur stable matrix. For stability analysis, we may calculate the characteristic polynomial $p_{2}(\lambda)=$ $\operatorname{det}\left(\lambda I-J_{F}\right)$ where $I$ is an identity matrix with appropriate dimensions. Unfortunately, unless we make further assumptions on $K$, we were not able to determine the characteristic polynomial easily. But with special assumptions, one could obtain a form similar to the one given in (21). Furthermore, the approach presented here could be extended to higher order periods. Moreover, instead of finding the characteristic polynomial, one may try to find a gain matrix $K$ which yields $J_{F}$ given above Schur stable. This may require some computational procedure. Our preliminary research reveals that some periodic orbits which cannot be stabilized by classical DFC can be stabilized with the proposed approach. These points are still under investigation.

\section{SIMULATION RESULTS}

As a simulation example, we considered the coupled map lattices, which exhibit various interesting dynamical behaviours. We will use the following one dimensional unidirectionally coupled lattice system :

$$
\begin{aligned}
& x(k+1)=f(x(k))+\varepsilon(f(y(k))-f(x(k))), \\
& y(k+1)=f(y(k))+\varepsilon(f(x(k))-f(y(k))),
\end{aligned}
$$

where $f(\cdot)$ is the logistic map given by $f(z)=r z(1-$ $z), \varepsilon>0$ is the coupling constant. This system, with $r=4$ and $\varepsilon=0.8$ has a period 2 orbit $\Sigma_{2}=\left\{w_{1}^{*}, w_{2}^{*}\right\}$, where $w^{i *}=\left(\begin{array}{cc}x_{i}^{*} & y_{i}^{*}\end{array}\right)^{T}, i=1,2$ and $x_{1}^{*}=y_{1}^{*}=$ $0.90450849718747, x_{2}^{*}=y_{2}^{*}=0.34549150281253$. By using the results given in (Morgül, 2003), (Morgül, 2005a), it can be shown that this period 2 orbit cannot be stabilized by classical DFC. By utilizing (32), it can be shown that $\Sigma_{2}$ can be stabilized with the proposed scheme with the gain $K=\alpha I$ for $\alpha>1.56$. Some simulation results are given in Figures 1-3 for $\alpha=1.57$. In these simulations, initial conditions are chosen as $x(0)=0.5, y(0)=0.7, r=4, \varepsilon=0.8$. In Figure 1, we show $d\left(x(k), \Sigma_{2}\right)$ versus $k$, and as can be seen the decay is exponential. Figure 2 shows $x(k)$ versus $y(k)$ plot for $k \geq 400$. As can be seen, solutions converge to $\Sigma_{2}$. Finally Figure 3 shows $u_{1}(k)$ and $u_{2}(k)$ vs. k.

\section{CONCLUSION}

In this paper we considered the problem of stabilization of unstable periodic orbits for discrete-time chaotic systems. Our approach is related to that of (Vieira, and Lichtenberg, 1996) for $T=1$, however the form of our proposed control law is different and relation with the DFC is more obvious. Moreover, the extension to $T>1$ and to higher dimensional cases are 
novel. We show that for $T=1$, the proposed scheme does not have the inherent limitations of DFC and EDFC. Following a technique used in (Morgül, 2003), (Morgül, 2005a), (Morgül, 2009), we first constructed a map whose fixed points correspond to the periodic orbits of the uncontrolled system. Then we studied the stability of the proposed scheme by using the constructed map by using linearization. Then the stability problem is reduced to studying the Schur stability of the Jacobian of this map evaluated at the fixed point corresponding to the periodic orbit. We also presented some simulation results supporting our results.

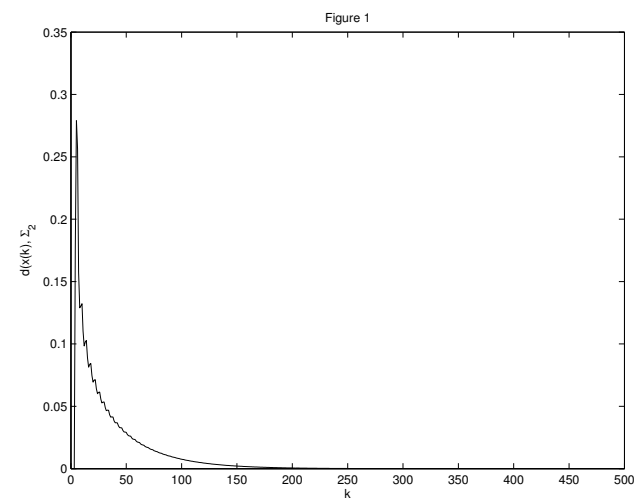

Fig. 1. $d\left(x(k), \Sigma_{2}\right)$ vs. $k$

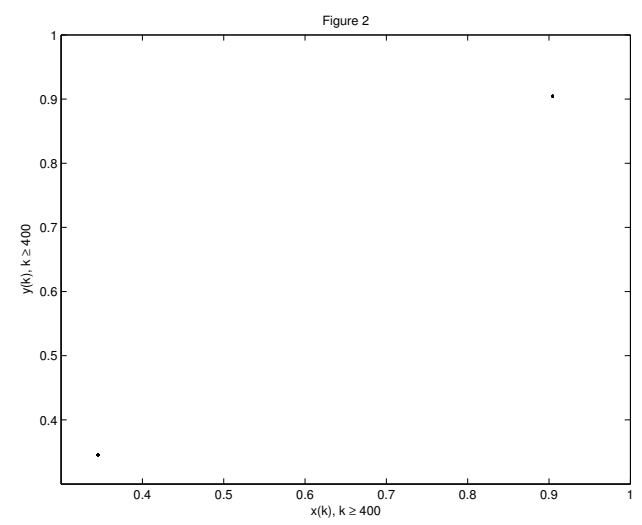

Fig. 2. $x(k)$ vs. $y(k)$ for $k \geq 400$

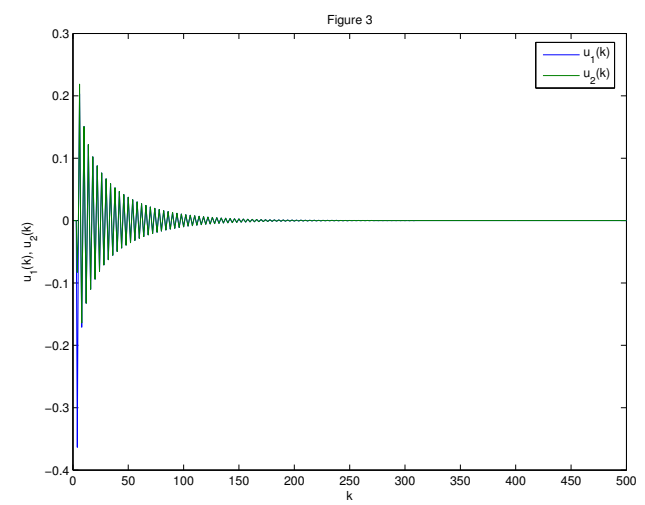

Fig. 3. $u_{1}(k)$ and $u_{1}(k)$ vs. $k$

\section{REFERENCES}

Bleich, M.E. , and Socolar, J.E.S. (1996), "Stability of periodic orbits controlled bt time delay feedback," Phys. Lett. A, 210, pp 87-94.

Chen, G., and X. Dong, From Chaos to Order : Methodologies, Perspectives and Applications (1999), World Scientific, Singapore.

Fiedler, B., Flunkert, V., Georgi, M., Hövel, P. and Schöll, E. (2007) "Refuting the odd-number limitation of time-delayed feedback control," Phys. Rev. Lett, 98, PRL No : 114110.

Khalil, H. K. (2002) Nonlinear Systems, 3rd ed. Prentice-Hall, Upper Saddle River.

Morgül, Ö. (2003) "On the stability of delayed feedback controllers," Phys. Lett. A314, 278-285.

Morgül, Ö. (2005a) "On the stability of delayed feedback controllers for discrete time systems," Phys. Lett. A335, 31-42.

Morgül, Ö. (2005b) "On the stabilization of periodic orbits for discrete time chaotic systems," Phys. Lett. A335, 127-138.

Morgül, Ö. (2006) "Stabilization of unstable periodic orbits for discrete time chaotic systems by using periodic feedback," Int. J. Bifurcation Chaos 16, 311-323.

Morgül, Ö. (2009) "A New Generalization of Delayed Feedback Control," Int. J. Bifurcation Chaos, 16, 365377.

Nakajima, H. (1997) "On analytical properties of delayed feedback control of chaos," Phys. Lett. A232, 207-210.

Ott, E., C. Grebogi, and J. A. Yorke (1990) "Controlling Chaos," Phys. Rev. Lett., 64, pp. 1196-1199.

Pyragas, K. (1992) "Continuous control of chaos by self-controlling feedback," Phys. Lett. A., 170, pp. 421-428.

Pyragas, K., (1995), "Control of chaos via extended delay feedback," Phys. Lett. A, 206, pp. 323-330.

Pyragas, K. (2001) "Control of chaos via an unstable delayed feedback controller," Phys. Rev. Lett., 86 pp. 2265-2268.

Socolar, J. E., Sukow, D. W., and Gauthier, D. J., (1994), "Stabilizing unstable periodic orbits in fast dynamical systems," Phys. Rev. E., vol. 50, pp. 32453248 .

Ushio, T. (1996) "Limitation of delayed feedback control in nonlinear discrete time systems," IEEE Trans. on Circ. Syst.- I 43, 815-816.

Vieira, d.S.M, \& Lichtenberg, A.J. (1996) "Controlling chaos using nonlinear feedback with delay," Phys. Rev. E54, 1200-1207. 